Review paper

\title{
Second re-irradiation: A delicate balance between safety and efficacy
}

\author{
Carsten Nieder ${ }^{\mathrm{a}, \mathrm{b}, *}$ \\ ${ }^{a}$ Department of Oncology and Palliative Medicine, Nordland Hospital Trust, 8092 Bodø, Norway \\ ${ }^{\mathrm{b}}$ Department of Clinical Medicine, Faculty of Health Sciences, UiT - The Artic University of Norway, 9038 Tromsø, Norway
}

A R T I C L E I N F O

\section{Keywords:}

Palliative radiotherapy

Re-irradiation

Toxicity

Recovery

\begin{abstract}
A B S T R A C T
Except for straightforward palliative indications such as painful bone metastases, re-irradiation is often characterized by a narrow therapeutic window and the potential for decreased efficacy and increased toxicity, especially if the cumulative total dose from both courses is high. Second re-irradiations tend to pose even bigger challenges and are thus offered in a restrictive manner to highly selected patients on a case-by-case basis. Normal tissue dose constraints are still an area of active investigation. Nevertheless, examples of potentially useful indications have been published. The present review briefly summarizes areas of uncertainty and opportunities for future research. If evidence-based concepts with acceptable side effect profiles can be developed, an increasing number of patients may benefit from additional radiotherapy to previously exposed target volumes.
\end{abstract}

\section{Introduction}

Radiation oncologists involved in palliative treatment scenarios frequently prescribe repeated courses of radiotherapy to anatomically distinct parts of the body, e.g., because of newly arising painful bone metastases. Due to non-overlapping dose distributions and moderate biologically equivalent doses, this type of retreatment is not particularly challenging. The complexity of decision-making and treatment planning increases when the new lesion is located close to the previous target volume, even in the absence of direct re-irradiation of an already treated lesion. For example, repeat radiosurgery (SRS) for new distant brain metastases or repeat stereotactic body radiotherapy (SBRT) for new lung lesions poses challenges regarding the cumulative dose to critical organs at risk, in case of lung SBRT mainly lung, esophagus, trachea, heart and large blood vessels [1,2]. Re-irradiation of the same target lesion is the most complex situation, however, some guidance from previous prospective studies and expert recommendations is available [3-6]. In contrast, very limited data exists to guide treatment planning for a second re-irradiation of the same target lesion [7]. These highly individualized treatments are uncommon, even in large institutions, thus rendering prospective studies very difficult to conduct. As recently reviewed, the literature is dominated by small retrospective studies that included heterogeneous patient populations, e.g. with brain metastases, spinal metastases, pelvic and thoracic targets. In the absence of widely agreed dose constraints, many assumptions have to be made when reviewing the dose distribution and signing the final plan. Therefore, the present review aims at proposing a strategy for collection of further clinical, and ideally also experimental, data, which may contribute to the development of evidence-based, safe dose/fractionation regimens.

\section{The clinical landscape}

Second re-irradiation comprises less than $1 \%$ of the workload in the author's current and former departments. The vast majority of these carefully selected patients receive treatment for metastatic bone lesions. However, we have recently also provided other examples of worthwhile re-re-irradiation to pelvic target volumes [8]. No serious toxicity was observed. One patient had sacral bone infiltration from rectal cancer and another patient lymph node and soft tissue recurrence from bladder cancer. In addition, patients with brain metastases may derive benefit from this approach, although the risk of radiation necrosis must be factored in $[9,10]$. We have previously reported a case of metastatic malignant melanoma initially treated with whole-brain radiotherapy (WBRT) in 2004, which was followed by SRS and repeated SRS to the same lesion in 2005 [11]. This patient is still alive as of summer 2018, free from radionecrosis and recurrence. Historically, re-re-irradiation has been employed with variable success in the era before effective systemic therapy became available and with now outdated equipment, e.g., for hematological and solid malignancies [12]. However, the available modern literature is still limited and definitive recommendations are difficult to develop [13-17]. Often, re-irradiation studies included a limited number of patients who were re-irradiated twice [18]. Since this subgroup was not the primary focus of the

\footnotetext{
* Address: Department of Oncology and Palliative Medicine, Nordland Hospital Trust, 8092 Bodø, Norway.

E-mail address: carsten.nieder@nlsh.no.
} 
publication, not all relevant data can be extracted, as already acknowledged in a previous review [7].

\section{The preclinical basis}

The re-irradiation tolerance or residual injury of different organs has been studied in animal models [19-21], as summarized in a comprehensive review [22]. However, a second re-irradiation has rarely been performed by these research groups. Chen and Hendry studied mouse skin injury after irradiation of the middle $3 \mathrm{~cm}$ of the tail [23]. They administered 25, 22.5, and $20 \mathrm{~Gy}$ with a time interval of 9 weeks (time required for skin healing) between these priming tolerance doses. Nine weeks after the third priming dose an additional test single dose was given. Among other endpoints, dose-response curves for healing were evaluated. Summarized briefly, residual injury was characterized by a $35 \%$ reduction in the iso-effective dose after three priming doses. This animal model does not closely resemble the clinical scenarios of rere-irradiation and is limited to skin only. Therefore, decisions have to be based on the magnitude of tissue recovery between the first and second course, and the assumption that the fundamental principles of radiobiology and damage repair in cells and tissues remain valid also after the second course. Ideally, additional experiments, e.g. for spinal cord, lung and bowel tolerance, would be performed to confirm the "disappearance" of large parts of the subclinical damage induced by both previous treatment courses, even if such experiments are time and resource consuming. Since kidney, heart and bladder have been shown to tolerate re-irradiation poorly (no recovery; in case of bladder for whole-organ irradiation, which is uncommon in the clinic) these organs should be considered critical organs at risk during treatment planning in patients.

\section{The magnitude of recovery from initial subclinical radiation effects}

The spinal cord data published by Ang et al. suggest that for a time interval of 1,2 , and 3 years between the treatment courses, cumulative doses of $150 \%, 156 \%$, and $167 \%$ of the first-line setting's tolerance dose appear possible [19]. Clinical data from patients who received two and even three courses of radiotherapy to the spine confirm the feasibility of re-irradiation with cumulative doses higher than first-line tolerance, albeit with limited follow-up due to the palliative nature of treatment $[24,25]$. Many clinicians have adopted the tolerance doses derived from the Ang et al. data. For example, Abusaris et al. set the maximum reirradiation dose as $50 \%$ more than the normal constraint if the interval was at least 12 months, also for re-re-irradiation [26]. For spinal cord, this resulted in an equivalent dose in 2-Gy fractions (EQD2) of $75 \mathrm{~Gy}$ for the second course. A dose adjustment of $25 \%$ was made for intervals between 6 and 12 months. Fig. 1 illustrates this approach (blue data points). Given that biological processes tend to proceed in a more continuous way, it appears possible that the orange data points may better describe the increase of tolerance doses over time. This hypothesis is accessible for testing in experimental and clinical settings. So far, the clinical toxicity data $[7,8,24,25,26]$ is fully compatible with the proposed $50 \%$ increase for time intervals $\geq 12$ months. It is not recommended to assume $100 \%$ recovery at any longer interval. It can not be advocated to adopt a time-dependent $25-50 \%$ dose increase to the heart [21]. Compared to simple models the clinical situation is complicated by additional risk factors for toxicity, e.g., comorbidity and different other oncological treatments. It is recommended to refrain from re-irradiation if prior radiotherapy was poorly tolerated and/or late tissue damage has been detected. This policy introduces an additional safety margin, because the population-based tolerance dose recommendations $\left(\mathrm{TD}_{5 / 5}\right.$ etc.) capture many events that occur in the part of the population that tolerated treatment less well than the majority. Excluding these patients, e.g. with severe skin and mucosal changes, from re-irradiation allows for prescribing higher doses with higher tumor control probability. Recently, retrospective efforts were made to decipher the tolerance of the brachial plexus and aorta to re-irradiation $[27,28]$. These clinical data ( $n=43$ patients) suggested that cumulative maximum dose to the plexus should be lower than the median ( $95 \mathrm{~Gy}$ ) and that re-irradiation within two years increased the risk of neuropathy. Based on time interval and cumulative dose, three risk groups were proposed [27]. For the thoracic aorta, maximum dose to $1 \mathrm{ml}$ should be lower than $120 \mathrm{~Gy}$ [28]. Independent validation of these predictive models is urgently awaited. In this context one has to be aware of the uncertainty around true cumulative dose assessment. Even with co-registration of different plans and deformable image registration the treatment planning scans only represent a snap-shot, which does not account for patient movement and organ movement during a course of fractionated radiotherapy. This uncertainty and "geographical miss" of organs at risk might be disadvantageous if the high-dose region unintentionally moves towards a dose-limiting structure. However, it might also protect such structures because of a blurring of the high-dose region over a larger area than visible on the snap-shot scan. Daily registration of the actually delivered dose would be required to definitely establish tolerance doses. For organs where parameters other than maximum dose are important (mean dose, V30, V50, or combinations of different dose-volume histogram metrics) a clarification is needed as to whether the same policy ( $50 \%$ more dose after $\geq 12$ months) should be recommended. It also appears relevant to study the influence of target volume size on development of toxicity. For example, if pelvic rere-irradiation involves one course with a large target volume, such as bilateral elective lymph node regions, the inclusion of the vasculature might result in a higher risk of reduced blood perfusion and tissue necrosis compared to three courses to small target volumes, especially if full overlap can be avoided. In head and neck cancer re-irradiation, published studies often contained some patients with recurrent tumors and others with second malignancies. The sparse literature about second re-irradiation so far does not contain sufficiently many patients with second malignancies to allow for meaningful analysis. In the uncommon situation of potential long-term survival one may consider efforts that limit the second cancer risk from re-irradiation [29-31].

\section{Perspective}

Even if second re-irradiation is a challenging topic that receives less attention than other recent developments, the available evidence and the experience at the author's department suggest that this strategy, which is inexpensive compared to other oncological treatments [32], may be effective in terms of symptom palliation, e.g. pain from bone metastases or soft tissue/lymph node tumors [8-10,24-26]. However, the therapeutic window is narrow and several studies have reported clinically relevant, sometimes fatal side effects such as soft tissue necrosis and fistula $[33,34]$. In order to improve the safety and reduce barriers for routine clinical administration (including legal aspects) a well-defined strategy is needed. The existing literature is difficult to put in context due to lack of comprehensive dosimetric and volumetric information. Only if individual institutions collaborate will we be able to establish sufficiently large databases and to recruit patients into prospective trials that can pave the way towards evidence-based treatment regimens, as already demonstrated for the first re-irradiation setting [35-37]. Both, clinical and experimental research should be conducted to deepen our understanding of tissue recovery and dosevolume effects. In the future, population-based dose constraints might be replaced or supplemented by personalized information based on innovative prediction models $[38,39]$. Cooperative groups should consider establishing expert panels, which review and modify the re-reirradiation concepts and plans of participating institutions comparable to pre-treatment quality assurance in large, landmark clinical trials. Outcomes of these "consensus" treatments should then be registered and monitored to increase the knowledge base and bridge the gap until formal prospective trials can be completed. 


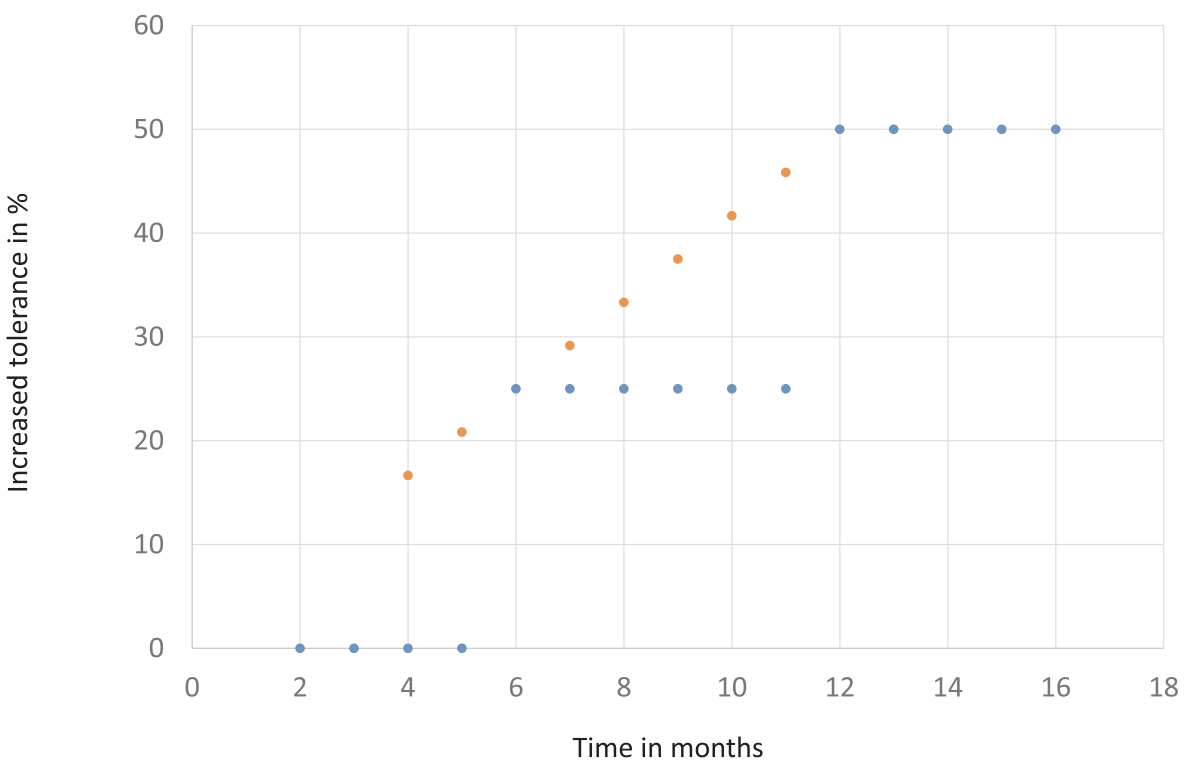

Fig. 1. Recovery and increased tolerance during the time interval after previous radiotherapy. The blue bullets illustrate the strategy published in [26]. If recovery is a continuous biological process, tolerance might increase in the manner illustrated by the orange bullets. (For interpretation of the references to colour in this figure legend, the reader is referred to the web version of this article.)
This research did not receive any specific grant from funding agencies in the public, commercial, or not-for-profit sectors.

\section{Declarations of interest}

None.

\section{References}

[1] Horne ZD, Dohopolski MJ, Clump DA, Burton SA, Heron DE. Thoracic reirradiation with SBRT for residual/recurrent and new primary NSCLC within or immediately adjacent to a prior high-dose radiation field. Pract Radiat Oncol 2018;8:e117-23.

[2] De Ruysscher D, Faivre-Finn C, Le Pechoux C, Peeters S, Belderbos J. High-dose reirradiation following radical radiotherapy for non-small-cell lung cancer. Lancet Oncol 2014;15:e620-4.

[3] Nieder C, Langendijk JA, Guckenberger M, Grosu AL. Prospective randomized clinical studies involving reirradiation: lessons learned. Strahlenther Onkol 2016;192:679-86.

[4] Krauze AV, Attia A, Braunstein S, Chan M, Combs SE, Fietkau R, et al. Expert consensus on re-irradiation for recurrent glioma. Radiat Oncol 2017;12:194. Erratum in: Radiat Oncol 2018;13:8.

[5] Harms W, Budach W, Dunst J, Cancer Breast, et al. Expert Panel of the German Society of Radiation Oncology (DEGRO). DEGRO practical guidelines for radiotherapy of breast cancer VI: therapy of locoregional breast cancer recurrences. Strahlenther Onkol 2016;192:199-208.

[6] Nieder C, De Ruysscher D, Gaspar LE, Guckenberger M, Mehta MP, Cheung P, et al. Reirradiation of recurrent node-positive non-small cell lung cancer after previous stereotactic radiotherapy for stage I disease: a multi-institutional treatment recommendation. Strahlenther Onkol 2017;193:515-24.

[7] Nieder C, Langendijk JA, Guckenberger M, Grosu AL. Second re-irradiation: a narrative review of the available clinical data. Acta Oncol 2018;57:305-10.

[8] Nieder C, Yobuta R, Mannsåker B. Second re-irradiation: clinical examples of worthwhile treatment. Cureus 2018;10:e2813.

[9] Balermpas P, Stera S, Müller von der Grün J, Loutfi-Krauss B, Forster MT, Wagner M, et al. Repeated in-field radiosurgery for locally recurrent brain metastases: feasibility, results and survival in a heavily treated patient cohort. PLoS One 2018;13:e0198692.

[10] Moreau J, Khalil T, Dupic G, Chautard E, Lemaire JJ, Magnier F, et al. Second course of stereotactic radiosurgery for locally recurrent brain metastases: safety and efficacy. PLoS One 2018;13:e0195608.

[11] Dalhaug A, Pawinski A, Norum J, Nieder C. An uncommon presentation and course of metastatic malignant melanoma: a case report. J Med Case Rep 2007;1:151.

[12] Nieder C, Langendijk JA, Guckenberger M, Grosu AL. Preserving the legacy of re irradiation: a narrative review of historical publications. Adv Radiat Oncol 2017;2:176-82.

[13] Dörr W, Gabryś D. The principles and practice of re-irradiation in clinical oncology: an overview. Clin Oncol (R Coll Radiol) 2018;30:67-72.

[14] Ho ALK, Jena R. Re-irradiation in the brain: primary gliomas. Clin Oncol (R Coll Radiol) 2018;30:124-36.

[15] Sadozye AH. Re-irradiation in gynaecological malignancies: a review. Clin Oncol ( $R$ Coll Radiol) 2018;30:110-5.

[16] Owens R, Muirhead R. External beam re-irradiation in rectal cancer. Clin Oncol (R Coll Radiol) 2018;30:116-23.
[17] Rulach R, Hanna GG, Franks K, McAleese J, Harrow S. Re-irradiation for locally recurrent lung cancer: evidence, risks and benefits. Clin Oncol (R Coll Radiol) 2018;30:101-9.

[18] Notter M, Piazena H, Vaupel P. Hypofractionated re-irradiation of large-sized recurrent breast cancer with thermography-controlled, contact-free water-filtered infra-red-A hyperthermia: a retrospective study of 73 patients. Int J Hyperthermia 2016;28:1-10.

[19] Ang KK, Jiang GL, Feng Y, et al. Extent and kinetics of recovery of occult spinal cord injury. Int J Radiat Oncol Biol Phys 2001;50:1013-20.

[20] Medin PM, Foster RD, van der Kogel AJ, et al. Spinal cord tolerance to reirradiation with single-fraction radiosurgery: a swine model. Int J Radiat Oncol Biol Phys 2012;83:1031-7.

[21] Wondergem J, van Ravels FJ, Reijnart IW, Strootman EG. Reirradiation tolerance of the rat heart. Int J Radiat Oncol Biol Phys 1996;36:811-9.

[22] Nieder C, Milas L, Ang KK. Tissue tolerance to reirradiation. Semin Radiat Oncol 2000;10:200-9.

[23] Chen FD, Hendry JH. Residual skin injury after repeated irradiation: differences observed using healing, macrocolony, and microcolony endpoints. Int J Radiat Oncol Biol Phys 1988;15:943-8.

[24] Thibault I, Campbell M, Tseng CL, Atenafu EG, Letourneau D, Yu E, et al. Salvage stereotactic body radiotherapy (SBRT) following in-field failure of initial SBRT for spinal metastases. Int J Radiat Oncol Biol Phys 2015;93:353-60.

[25] Katsoulakis E, Riaz N, Cox B, Mechalakos J, Zatcky J, Bilsky M, et al. Delivering a third course of radiation to spine metastases using image-guided, intensity-modulated radiation therapy. J Neurosurg Spine 2013;18:63-8.

[26] Abusaris H, Storchi PR, Brandwijk RP, Nuyttens JJ. Second re-irradiation: efficacy, dose and toxicity in patients who received three courses of radiotherapy with overlapping fields. Radiother Oncol 2011;99:235-9.

[27] Chen AM, Yoshizaki T, Velez MA, Mikaeilian AG, Hsu S, Cao M. Tolerance of the brachial plexus to high-dose reirradiation. Int J Radiat Oncol Biol Phys 2017;98:83-90.

[28] Evans JD, Gomez DR, Amini A, Rebueno N, Allen PK, Martel MK, et al. Aortic dose constraints when reirradiating thoracic tumors. Radiother Oncol 2013;106:327-32.

[29] Marcu LG. Photons - radiobiological issues related to the risk of second malignancies. Phys Med 2017;42:213-20.

[30] Trott KR. Special radiobiological features of second cancer risk after particle radiotherapy. Phys Med 2017;42:221-7.

[31] Bezak E, Takam R, Yeoh E, Marcu LG. The risk of second primary cancers due to peripheral photon and neutron doses received during prostate cancer external beam radiation therapy. Phys Med 2017;42:253-8.

[32] Nguyen TK, Goodman CD, Boldt RG, Warner A, Palma DA, Rodrigues GB, et al. Evaluation of health economics in radiation oncology: a systematic review. Int $J$ Radiat Oncol Biol Phys 2016;94:1006-14.

[33] Feddock J, Cheek D, Steber C, Edwards J, Slone S, Luo W, et al. Reirradiation using permanent interstitial brachytherapy: a potentially durable technique for salvaging recurrent pelvic malignancies. Int J Radiat Oncol Biol Phys 2017;99:1225-33.

[34] Peulen H, Karlsson K, Lindberg K, Tullgren O, Baumann P, Lax I, et al. Toxicity after reirradiation of pulmonary tumours with stereotactic body radiotherapy. Radiother Oncol 2011;101:260-6.

[35] Langer CJ, Harris J, Horwitz EM, Nicolaou N, Kies M, Curran W, et al. Phase II study of low-dose paclitaxel and cisplatin in combination with split-course concomitant twice-daily reirradiation in recurrent squamous cell carcinoma of the head and neck: results of Radiation Therapy Oncology Group Protocol 9911. J Clin Oncol 2007;25:4800-5.

[36] Niyazi M, Adeberg S, Kaul D, Boulesteix AL, Bougatf N, Fleischmann DF, et al. Independent validation of a new reirradiation risk score (RRRS) for glioma patients 
predicting post-recurrence survival: a multicenter DKTK/ROG analysis. Radiother Oncol 2018;127:121-7.

[37] Arthur DW, Winter KA, Kuerer HM, Haffty BG, Cuttino LW, Todor DA, et al. NRC Oncology-Radiation Therapy Oncology Group Study 1014: 1-Year toxicity report from a phase 2 study of repeat breast-preserving surgery and 3-dimensional conformal partial-breast reirradiation for in-breast recurrence. Int J Radiat Oncol Biol
Phys 2017;98:1028-35.

[38] Kerns SL, West CM, Andreassen CN, Barnett GC, Bentzen SM, Burnet NG, et al. Radiogenomics: the search for genetic predictors of radiotherapy response. Future Oncol 2014;10:2391-406.

[39] Flux GD, O'Sullivan J, Gaze MN, Prise KM. Opportunities for research in molecular radiotherapy. Br J Radiol 2017;90:20160921. 